\title{
Un intento de considerar la escorrentía muy rápida (emr) en la modelación hidrológica
}

Roberto Fredy Ávalos Lingán*

\section{RESUMEN}

En el Manual de Referencias Hidrológicas para el Diseño de Obras de Drenaje Menor se presenta información valiosa de intensidades máximas de lluvia para todo el territorio hondureño y de pruebas de infiltraciones realizadas en diferentes tipos de suelos, donde para el caso de las intensidades de 5 minutos, el $77 \%$ de las estaciones sobrepasan fácilmente los $100 \mathrm{~mm} / \mathrm{h}$ (intensidades fuertes) y el $60 \%$ de la pruebas de infiltración muestran una infiltración inicial que exceden los $100 \mathrm{~mm} / \mathrm{h}$. La disponibilidad de esta información y, sobre todo sus magnitudes, nos ha permitido hacer realidad la idea de hace varios años de intentar hacer uso de dicha información para ser incluida en un modelo hidrológico y representar la Escorrentía Muy Rápida (EMR); es decir, cuando la intensidad de lluvia es mayor que la capacidad de infiltración del suelo.

Como primer ensayo se introdujo en un modelo hidrológico conceptual una simple función lineal para representar la EMR, dependiendo ésta de las intensidades máximas de lluvia de 5 min, de la capacidad máxima de infiltración en 5 min y de la lluvia en $\mathrm{mm} / \mathrm{día}$, observándose que a una misma lluvia el aumento de la EMR depende del aumento del parámetro de escurrimiento muy rápido (CMR), parámetro que gobierna la función.

Palabras clave:Intensidades máximas de la lluvia, Capacidad máxima de la infiltración, Infiltración inicial, Escorrentía muy rápida.

\section{ABSTRACT}

Hydrological Reference Manual for the Design of Drainage Minor Works, published by the FHIS valuable information is presented maximum intensities of rainfall for the entire Honduran and infiltration tests conducted in different soil types. Where in the case of the maximum intensity of 5 minutes $77 \%$ of the stations easily exceed 100 $\mathrm{mm} / \mathrm{h}$ (strong intensities) and $60 \%$ of the infiltration tests show an initial infiltration

“Universidad Nacional Autónoma de Honduras. Facultad de Ingeniería, Escuela de Ingeniería Civil. 
exceeding $100 \mathrm{~mm} / \mathrm{h}$. The availability of this information and especially their magnitudes allowed us to realize the idea several years ago attempting to use this information to be included in a hydrologic model and represent the very rapid runoff (EMR), is to say when rainfall intensity is higher than soil infiltration capacity.

As a first test was introduced in a conceptual hydrological model a simple linear function to represent the EMR. Depending this fonction, of the rainfall intensities of 5 $\mathrm{min}$, of the infiltration capacity in $5 \mathrm{~min}$ and the rain in $\mathrm{mm} /$ day. Observing that the same rain increased EMR dependent increase in CMR ratio, a parameter that governs the function.

Key words: Maximum rain Intensity, Maxima Capacity of infiltration initial, runoff very fast. 


\section{INTRODUCCIÓN}

En países de clima tropical como en Honduras la intensidad máximas de lluvia son acentuadas. Esto lo muestra la información observada de la red nacional de estaciones (medidoras de esta variable), específicamente la correspondiente a 5 minutos de duración, donde el $77 \%$ de las estaciones sobrepasan fácilmente los $100 \mathrm{~mm} / \mathrm{h}^{1}$. En el Manual de Referencias Hidrológicas para el Diseño de Obras de Drenaje Menor existen datos de pruebas de infiltración en diferentes condiciones de suelos realizadas en distintas partes del país; de dicha fuente se obtiene que en el 60 $\%$ de las pruebas la infiltración inicial sobrepasa los $100 \mathrm{~mm} / \mathrm{h}$.

La disponibilidad de esta información, y sobre todo sus magnitudes, nos ha permitido hacer realidad la idea de hace varios años de intentar hacer uso de dicha información para ser incluida en un modelo hidrológico y representar las escorrentías muy rápidas. Sabemos que en una cuenca comúnmente hay diferentes tipos de suelo, vegetación, orografía y clima, factores que influencian en el comportamiento de variables como la infiltración del suelo y la intensidad de la lluvia. A pesar de esto, la idea siempre ha sido su utilización en modelos hidrológicos conceptuales.

El objetivo principal de esta investigación exploratoria es introducir en un modelo hidrológico conceptual la Escorrentía Muy Rápida (EMR), representada por la función de la intensidad máxima $(\mathrm{mm} / \mathrm{h})$ de 5 minutos y de la capacidad máxima de infiltración del suelo ( $\mathrm{mm} / \mathrm{h}$ ), también a los 5 minutos y, además, observar de manera muy simple su influencia en los resultados de la calibración.

\section{INFORMACIÓN BASE}

Para llevar a bien esta investigación, se consideró, para el caso de la lluvia, las magnitudes de las intensidades máximas de 5 minutos en $\mathrm{mm} / \mathrm{h}$; y para los suelos, las capacidades máximas de infiltración en 5 minutos en $\mathrm{mm} / \mathrm{h}$ que fue representada por la infiltración inicial fo². La Figura 1 muestra las intensidades máximas de la lluvia para las diferentes estaciones medidoras y la Figura 2 las infiltraciones iniciales de diferentes tipos de suelos en Honduras. La información presentada en ambas figuras (Figura 1 y 2) servirá para ubicarnos en cuanto a las magnitudes de dichas variables.

\footnotetext{
${ }^{1}$ Información obtenida del Manual de Referencias Hidrológicas para el Diseño de Obras de Drenaje Menor. ${ }^{2}$ ídem
} 
Figura 1: Intensidades máximas de la lluvia en 5 minutos en $\mathrm{mm} / \mathrm{h}$ para las estaciones de Honduras

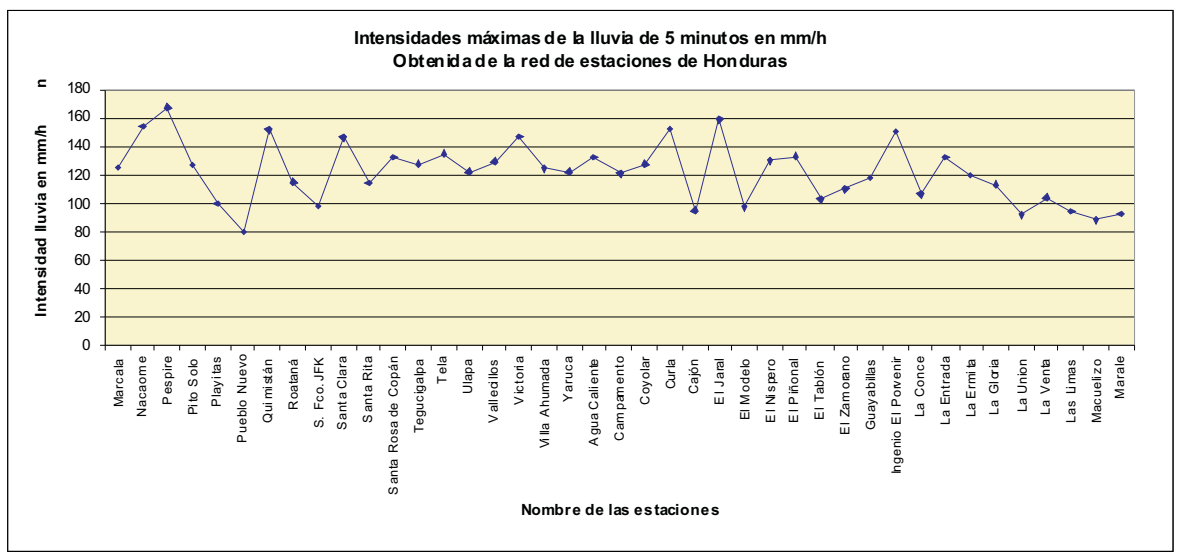

Figura 2: Infiltración inicia Fo en $5 \mathrm{~min}(\mathrm{~mm} / \mathrm{h})$ para diferentes suelos de Honduras

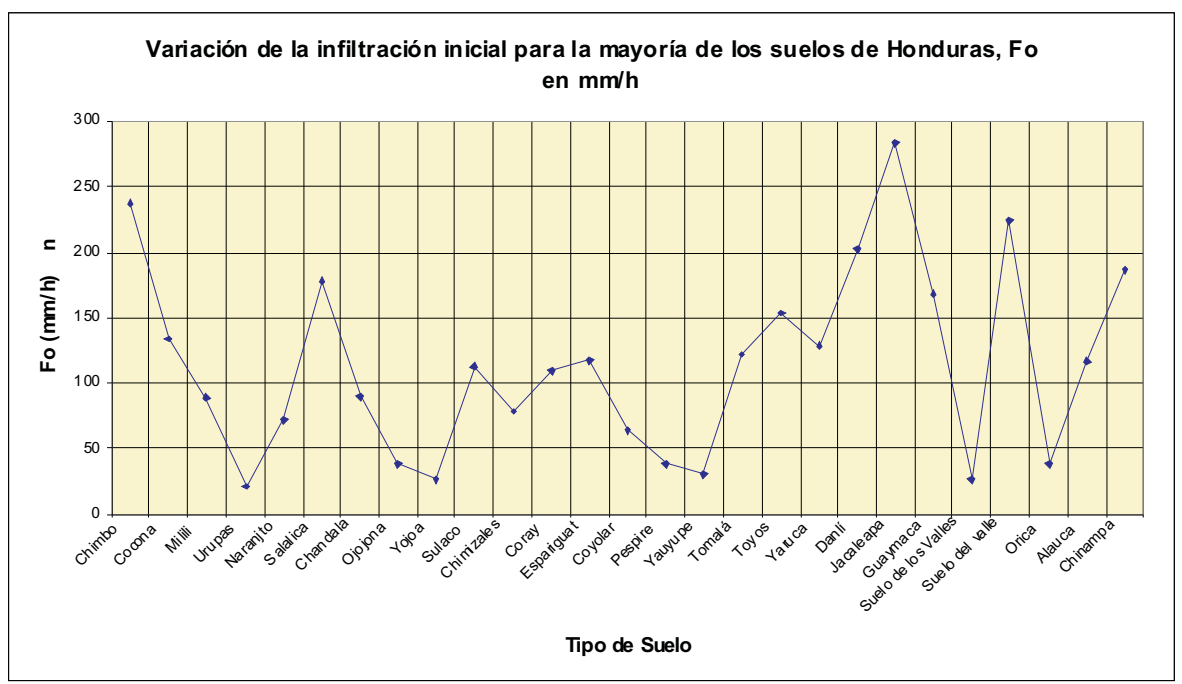

\section{PROCEDIMIENTO}

Los modelos hidrológicos conceptuales no son específicamente modelos físicos medibles, sino más bien se fundamentan en funciones matemáticas gobernadas por parámetros para describir los procesos hidrológicos ocurridos en la cuenca hidrográfica. El procedimiento de calibración de los parámetros del modelo permite 
llegar a acercarse y a simular las magnitudes de las variables observadas de dichos procesos hidrológicos. Por supuesto que esta simulación será más exitosa cuando se disponga de variables observadas que representen realmente las magnitudes de dicho proceso hidrológico tanto en el tiempo como en el espacio. De no ser así, aunque se aplique el mejor modelo, donde el usuario reduzca al mínimo o estire al máximo los parámetros, no se llegará a alcanzar buenos resultados. Para el caso que nos concierne hemos considerado que la Escorrentía Muy Rápida (EMR) es función lineal de:

$$
E M R=f(I N T, I N F, P)
$$

Donde: EMR Representala escorrentía muy rápida en $\mathrm{mm} / \mathrm{día}$

INT Representala intensidad máxima de la lluvia de 5 minutos en $\mathrm{mm} / \mathrm{h}$.

INF Representa la capacidad máxima de infiltración del suelo en 5 minutos y en $\mathrm{mm} / \mathrm{h}$. En este caso es representada por la infiltración inicial Fo.

P Representa la lluvia diaria en $\mathrm{mm}$

Existirá escorrentía muy rápida cuando INT sea mayor que INF.

\section{APLICACIÓNYRESULTADOS}

La Figura 3 presenta la aplicación de un ensayo de lo que podría ser el comportamiento de la Escorrentía Muy Rápida en función de las variables ya mencionadas.

\section{Figura 3: Comportamiento de la Escorrentía Muy Rápida (EMR)}

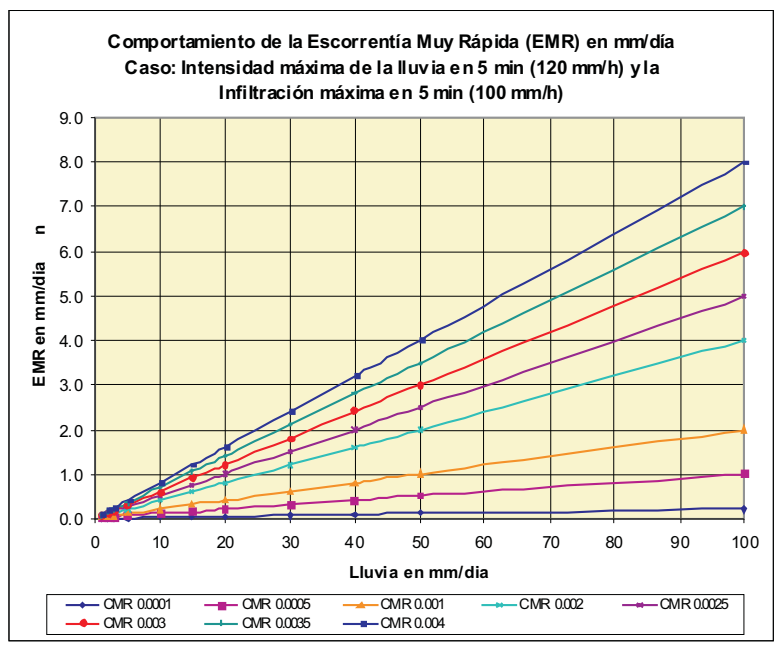


CMR son los valores del parámetro que gobierna la función para determinar la Escorrentía Muy Rápida. Mediante el uso de un modelo hidrológico conceptual se aplicó este concepto de Escorrentía Muy Rápida con el fin de observar de manera sencilla la influencia de considerar en la modelación hidrológica dicho concepto.

En esta aplicación se hizo variar únicamente la intensidad de la lluvia de 100 a 200 $\mathrm{mm} / \mathrm{h}$; todo el resto del modelo permaneció igual. La Figuras 4 al 7 muestran la aplicación mencionada.

Figura 4: Sensibilidad del parámetro relación Intensidad de la lluvia máxima en 5 minutos $(100 \mathrm{~mm} / \mathrm{h})$ con la Infiltración máxima en 5 minutos $(100 \mathrm{~mm} / \mathrm{h})$

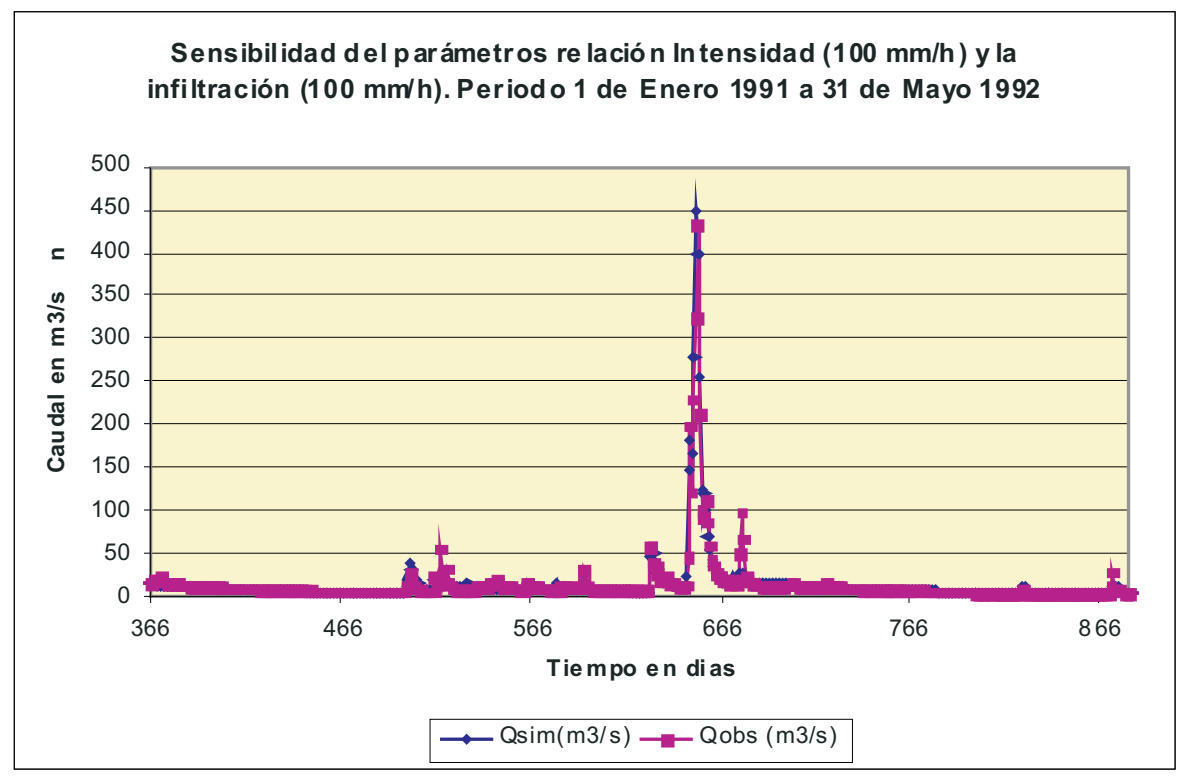

De dicho grupo de figuras, la figura 5 muestra el inicio de la influencia de EMR, donde aparece un aumento de los caudales picos; pero a su vez se muestra una disminución en la parte baja del hidrograma.

Esto es normal porque el resto de los parámetros del modelo no han sido tocados. Más notorio es en la figura 7, donde se considera una intensidad de lluvia de 200 $\mathrm{mm} / \mathrm{h}$. 
Figura 5: Sensibilidad del parámetro relación Intensidad de la lluvia máxima en 5 minutos $(120 \mathrm{~mm} / \mathrm{h})$ con la Infiltración máxima en 5 minutos $(100 \mathrm{~mm} / \mathrm{h})$

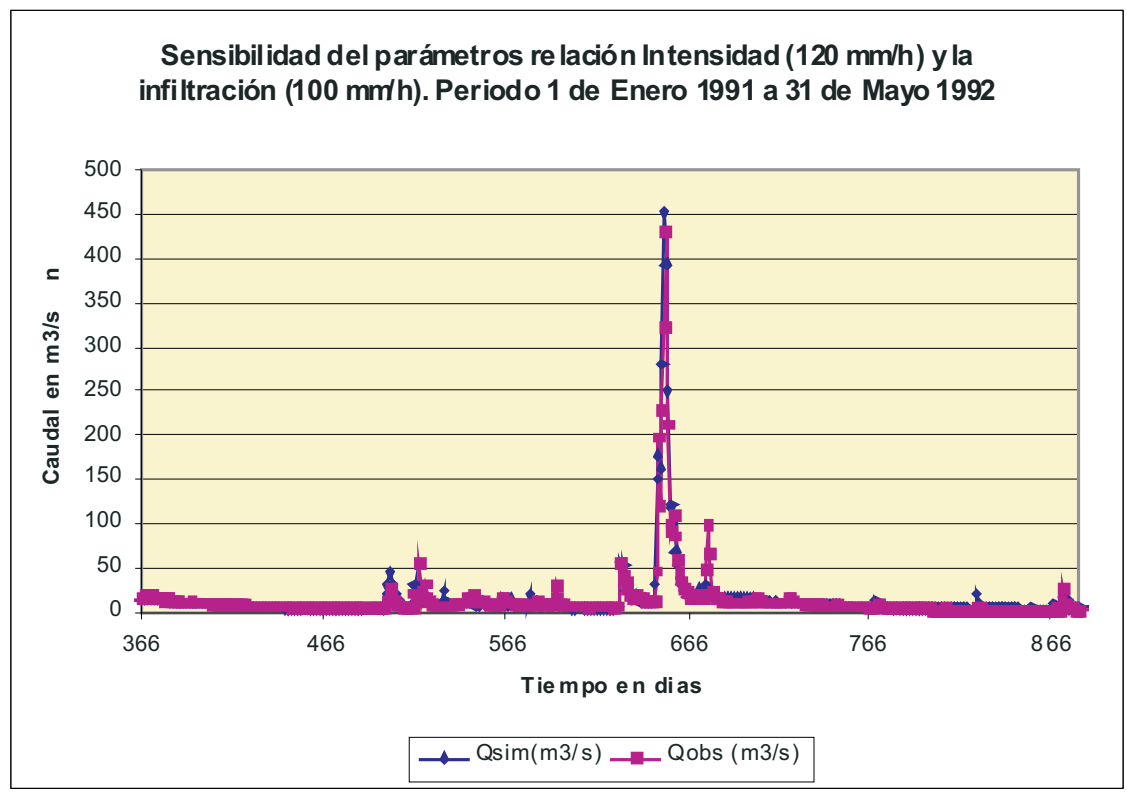

Figura 6: Sensibilidad del parámetro relación Intensidad de la lluvia máxima en 5 minutos $(150 \mathrm{~mm} / \mathrm{h}$ ) con la infiltración máxima en 5 minutos $(100 \mathrm{~mm} / \mathrm{h})$

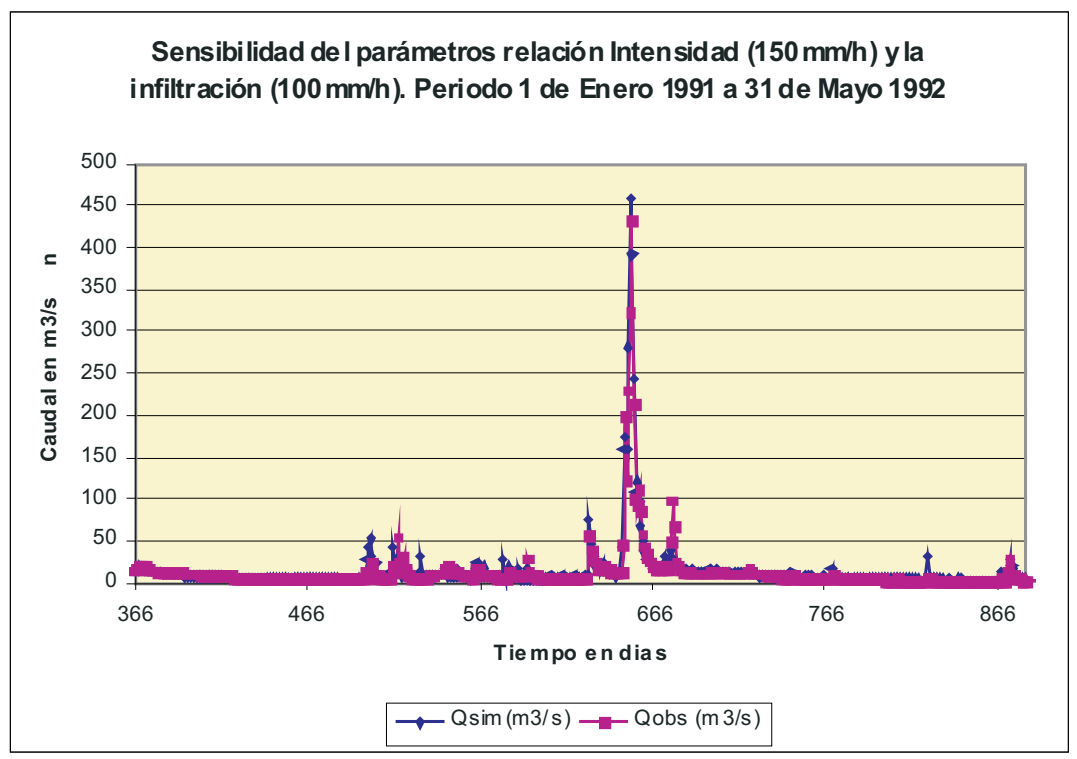


Figura 7: Sensibilidad del parámetro relación Intensidad de la lluvia máxima en 5 minutos $(200 \mathrm{~mm} / \mathrm{h})$ con la infiltración máxima en 5 minutos $(100 \mathrm{~mm} / \mathrm{h})$

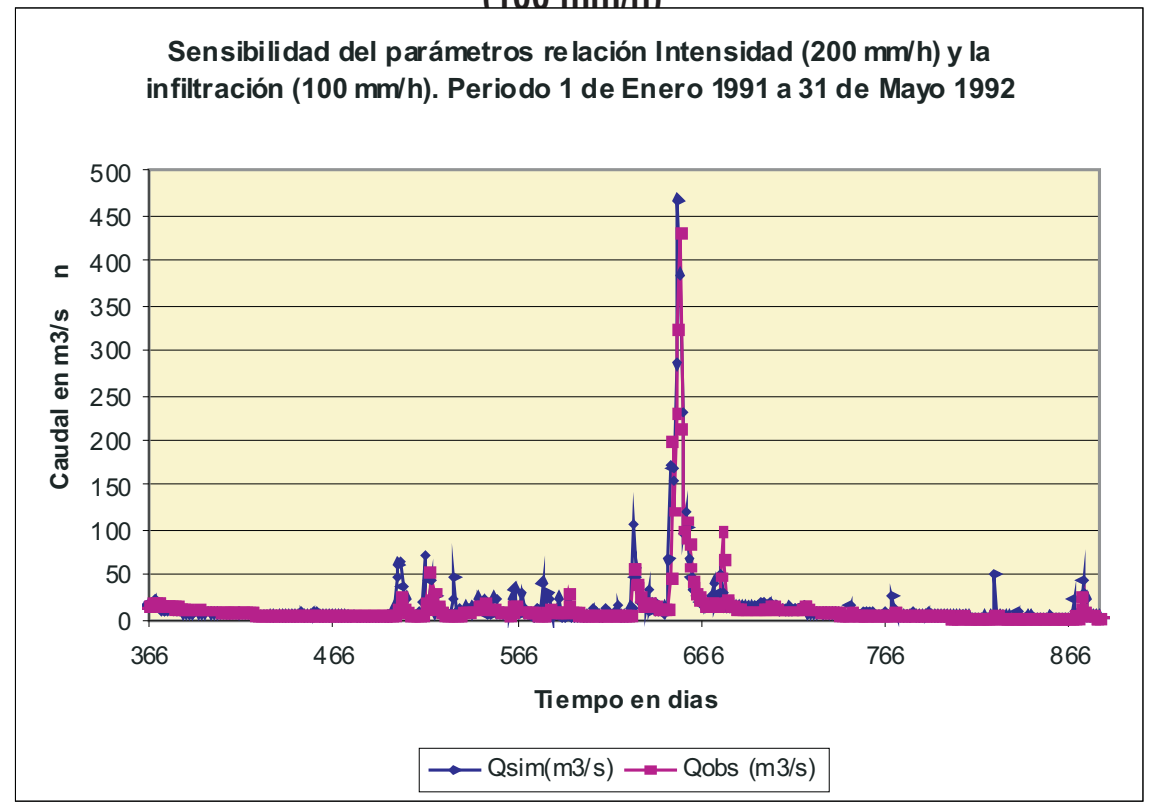

\section{CONCLUSIÓN}

El proceso de intensidad máxima de la lluvia en la cuenca y su relación con la capacidad de infiltración de suelo es complejo para poder definir la participación de un flujo muy rápido. Posiblemente se pueda establecer funciones más integrales. En esta etapa de la investigación y de la sencillez como se ha planteado, pensamos que es un buen intento para mostrar la presencia y su influencia en los hidrogramas que simulan los modelos hidrológicos conceptuales.

De la presente investigación se concluye lo siguiente: De acuerdo a los conceptos planteados se observa que a una misma lluvia ( $\mathrm{mm} / \mathrm{día})$ el aumento de la Escorrentía Muy Rápida depende del aumento del CMR.

El considerar una función lineal para conceptualizar el proceso de la escorrentía muy rápida provoca un aumento de los picos de los hidrogramas. 


\section{RECOMENDACIÓN}

Continuar con la investigación de la escorrentía muy rápida en el caso de cuencas donde la intensidad máxima de la lluvia es fuerte y establecer nuevas funciones y conceptualizaciones del proceso. Posiblemente sea necesario probar con diferentes tipos de modelos hidrológicos conceptuales.

\section{BIBLIOGRAFÍA}

Ávalos Lingan, Roberto. (2009) Investigación del Modelaje Hidrológico. Modelo AVAMODEL. Tegucigalpa.

Bergstrom, Sten. (1970). Modelo HBV. Hydrological Bureau Waterbalance Division. Suecia.

Empresa Nacional de Energía Eléctrica (ENEE). Organismo facilitador de la información climática e hidrológica. Departamento de Recursos Hídricos.

Lundin, Lars-Christer. (2000). Sustainable Water Management in the Baltic Sea Basin. Book I, The Waterscape. Sweden. Uppsala University. 